\title{
The effects of preoperative neuromuscular electrical stimulation on the postoperative quadriceps muscle strength and functional status in patients with fast-track total knee arthroplasty
}

\author{
Raziye ŞAVKIN, Nihal BÜKER, Harun R. GÜNGÖR
}

From the Department of Orthopaedics and Traumatology, Pamukkale University, Denizli, Turkey

This study aimed to investigate the effect of preoperative neuromuscular electrical stimulation (NMES) on postoperative quadriceps muscle strength, functional status, and quality of life in patients with fast-track total knee arthroplasty (TKA).

This prospective study was carried out at Orthopedics department from September 2017 to October 2018. A total of 40 patients were randomly divided into NMES $(n=20)$ and control group $(n=20)$. Patients in NMES group were asked to use home NMES device daily for 20 minutes, 5 times a day, for 6 weeks before surgery. The control group was placed on the 6-week waiting list for surgery without any preoperative intervention. Standard home exercise program was applied to both groups after discharge. The patients were evaluated baseline, preoperatively (6-weeks after baseline) and at the 4th and 12th weeks after surgery. Knee range of motion, quadriceps muscle strength, patient-reported (WOMAC and KOOS) and performance-based activity limitation (30-second chair-stand test, 40-meter fast-paced walk test, and stair-climb test) were evaluated at each visit.

Preoperative NMES resulted in significant improvement in KOOS-function in daily living and WOMAC total score $(p \leq 0.05)$ but had a non-significant trend toward to improve quadriceps muscle strength, KOOS-pain and -other symptoms, performancebased activity limitation, and quality of life scores $(p>0.05)$. However, there was no significant difference between groups in the postoperative period $(p>0.05)$. NMES has beneficial effects in terms of patientreported and performance-based physical functions and quality of life in preoperative period; however, it does not provide any additional benefit for postoperative outcomes in patients with fast-track TKA.

Keywords : knee arthroplasty ; neuromuscular electrical stimulation ; fast-track ; physiotherapy ; patient-reported outcomes; quadriceps muscle strength.

\section{INTRODUCTION}

Knee osteoarthritis is a degenerative disease characterized by pain, joint stiffness, swelling, limitations of daily living activities and decreased quality of life. The prevalence of symptomatic and radiographic knee osteoarthritis is $37.4 \%$ and $12.1 \%$ respectively for adults $\geq 60$ years (1). Total knee arthroplasty (TKA) is a cost-effective

Raziye Şavkın',

- Nihal Büker ${ }^{1}$,

- Harun R. Güngör ${ }^{2}$.

${ }^{1}$ School of Physical Therapy and Rehabilitation, Pamukkale University, Denizli, Turkey.

${ }^{2}$ Orthopedics and Traumatology Department, Pamukkale University Medical Faculty, Denizli, Turkey.

Correspondence : Raziye Şavkın, School of Physical Therapy and Rehabilitation, 20070, Pamukkale University, Denizli, Turkey. Phone : +90 25829642 84, Fax: +90 2582964494. Email : raziyesavkin@hotmail.com

- 2021, Acta Orthopædica Belgica. 
and successful treatment option for patients who experience chronic, debilitating symptoms despite the exhaustion of all nonoperative and conservative treatment modalities. Current research in TKA focuses on shortening the length of hospital stay, reducing morbidity and complications, functional improvement, and highest patient satisfaction (2). In order to optimize this surgery, fast-track/enhanced recovery surgical protocols have been established as evidence-based, multidisciplinary and multimodal approaches $(3,4)$. The fast-track protocol aims to understand pathophysiology of the surgical stress response (endocrine, metabolic, immunological, and others), enhance recovery to restore functionality, and identify the predictors affecting the length of postoperative hospital stay (5).

The restoration of quadriceps strength and function following TKA is an essential component of successful surgical outcomes. Quadriceps muscle strength may decrease approximately $60-62 \%$ one month after TKA surgery (6), and this weakness may persist for up to 6-13 years postoperatively (7). Also, preoperative quadriceps strength may affect postoperative functionality (8). For these reasons, preoperative physiotherapy interventions have attracted interest from researchers. Neuromuscular electrical stimulation (NMES) is one of the therapeutic interventions to accelerate recovery and improve quadriceps function in patients undergoing TKA surgery. Despite many studies support the use of NMES following TKA surgery (9), there is limited understanding of the effectiveness of the preoperative use of NMES (10). However, researchers support the use of NMES before TKA surgery, but it is not evidence-based (11). Therefore, our study aimed to determine the effect of preoperative NMES application on postoperative quadriceps muscle strength, functional status, and quality of life outcomes in patients with fast-track TKA surgery.

\section{MATERIAL AND METHODS}

This single-center, prospective, randomized, single-blind study was conducted at Orthopedics and Traumatology Department of a University hospital. The study was approved by the Pamukkale
University Non-invasive Clinical Research Ethics Committee and an informed consent form was signed by all patients.

Inclusion criteria were as follows : aged 50-75, patients scheduled for unilateral TKA surgery due to primary osteoarthritis, ability to cooperate and understand verbal and written instructions. Exclusion criteria were as follows : revision TKA surgery, the ASA score $>3$, previous major orthopedic surgery in either lower extremities, neurologic disease, diagnosed psychiatric problems, regular hypnotic and/or anxiolytic medication usage, dementia, implanted pacemaker or defibrillator, dermatologic problems affecting lower extremity.

From September 2017 to October 2018, 157 patients registered to university hospital for TKA surgery. Patients who met the exclusion criteria $(n=87)$ and refused to participate in the study $(n=16)$ were excluded. At baseline, 54 patients ( 32 from NMES, 22 from control group) were evaluated, but 12 patients (7 from NMES, 5 from control group) were excluded because they declined surgery just before the preoperative evaluation. 52 patients underwent fast-track TKA surgery. Two patients from the control group did not participate the last evaluation. The final study sample consisted of 40 patients, with 20 in the NMES group and 20 in the control group.

Demographic (age, gender) and clinical data (body mass index, Modified Charlson comorbidity index, length of hospital stay, dominant and operated extremity) of patients were recorded. Evaluations were performed by a blinded investigator at baseline, preoperatively (after NMES application or 6-week surgical waiting period), weeks 4 and 12 postoperatively. Due to the increase in TKA surgery rates, a core set that complies with the International Functioning, Disability and Health Classification framework (12) and includes performance-based tests (recommended by OARSI) has been proposed (13). These studies were considered when determining the outcome measures. Knee range of motion (ROM) assessed with a digital goniometer (HALO Medical Devices, Australia), quadriceps muscle strength measured with a hand-held dynamometer (Commander Muscle Tester, J Tech, USA), patient-reported activity limitations 
determined by The Western Ontario and McMaster Universities Arthritis Index (WOMAC) and Knee injury and Osteoarthritis Outcome Score (KOOS), performance-based activity limitations evaluated with 30 -s chair-stand test, 40 m fast-paced walk test, and stair-climb test, and quality of life evaluation investigated with The World Health Organization Quality of Life Instrument, Short Form (WHOQOLBREF).

A portable four-channel digital electrical stimulator (QuadStar ${ }^{\circledR}$ II - Multi-Modality Stimulator, USA) was used for quadriceps muscle stimulation. Two $6 \times 9 \mathrm{~cm}$ electrodes were located at proximal and the distal part to the rectus femoris and vastus medialis muscles. Stimulation parameters were set as follow : biphasic, symmetrical, square waveform, frequency $50 \mathrm{~Hz}$, pulse duration $400 \mu \mathrm{s}$, ramp-up time $2 \mathrm{sec}$, contraction time $10 \mathrm{~s}$, ramp-down time $2 \mathrm{sec}$, rest time $30 \mathrm{~s}$, for 20 minutes, 5 days a week for 6 weeks. Patients were encouraged to use the stimulator at maximum tolerated high intensity with visible muscle contractions. The patient was placed in a sitting position, hip at $90^{\circ}$ flexion, knee at $60^{\circ}$ of flexion, the foot flat on the floor, and toes against a wall or ankle fastened with Velcro to permit isometric muscle contraction; patients contracted their quadriceps at each NMES stimulus. The NMES device was given to patients for home use, and patients were called at 2 -week intervals to encourage them to use the stimulator regularly. The control group was placed on the 6-week waiting list for surgery without any preoperative intervention.

All operations were performed by the same surgeon using the same brand and type of prosthesis. All patients received posterior stabilized fixed bearing TKA (NexGen Legacy ${ }^{\circledR}$ Posterior Stabilized Knee-Fixed Bearing, Zimmer-Biomet Inc., Warsaw, Indiana, USA), and high viscosity polymethyl methacrylate bone cement (Oliga-G21 srl-Via S.Pertini, San Possidonio [MO], Italy). The operation was performed through a medial parapatellar approach and without using tourniquet. The same institutional fast-track protocol was utilized on all patients.

The early postoperative physical therapy protocol focused on rapid mobilization under guidance of a physiotherapist. The patients were mobilized within
4 hours after surgery and a standard physical therapy program was performed during hospitalization (cold pack applied for 15 minutes every 2 hours, ankle pump exercises, quadriceps isometric exercises, active assistive heel slide exercise in bed and knee flexion exercise in sitting position -3 sets of 10 repetitions). The patients were discharged home when discharge criteria were met. A standard home-based exercise program was performed to all patients for 12 weeks in the postoperative period.

The number of patients included in the study was determined based on WOMAC data (14). The preoperative calculated sample size was 20 patients for each group with a power of $80 \%(\beta=0.2)$, and a statistical significance of $95 \%(\alpha=0.05, p=0.05)$. Therefore, we included 20 patients for the NMES group and 20 patients for the control group. According to WOMAC total score results, we had a very large effect size $(\mathrm{d}=1.4)$ and we reached $\% 99.7$ power with a $95 \%$ confidence interval.

Obtained data were analyzed by using the Statistical Package for Social Sciences (version 24 ; SPSS Inc., Chicago, IL). Continuous variables were given as mean \pm standard deviation, median (minimum and maximum), and categorical variable values are presented as absolute numbers and percentages. Categorical data were compared with use of the Chi-square $\left(\chi^{2}\right)$ test. The conformity of continuous variables with normal distribution was evaluated using the Shapiro-Wilk test. Independent Samples t-test for parametric test assumptions and Mann-Whitney U Test for non-parametric test assumptions were used for comparison of the groups. One-way repeated-measure ANOVA was used to compare the normally distributed data from the parameters repeatedly measured in the innergroup analysis, and Friedman analysis of variance was performed for the remaining data set. Statistical significance was set at $\mathrm{p} \leq 0.05$.

\section{RESULTS}

Demographic and clinical characteristics of the patients are given in Table I. There was no significant difference between the groups in terms of age, body mass index, Modified Charlson comorbidity index ( $p>0.05$ ). Length of hospital stay was $29.75 \pm 7.07$ 
Table I. - Patients' demographics

\begin{tabular}{|l|c|c|c|}
\hline & NMES Group $(\mathrm{n}=20)$ & Control Group $(\mathrm{n}=20)$ & $P$ Value \\
\hline Age (year) & $64.1 \pm 5.06(56-74)$ & $64.25 \pm 5.52(52-73)$ & 0.929 \\
\hline Body mass index $\left(\mathrm{kg} / \mathrm{m}^{2}\right)$ & $32.03 \pm 4.25(24.22-40)$ & $31.85 \pm 5.74(21.77-40)$ & 0.547 \\
\hline Modified Charlson Comorbidity Index & $3.3 \pm 0.98(1-5)$ & $2.9 \pm 1.12(1-5)$ & 0.242 \\
\hline Length of hospital stay (hours) & $29.75 \pm 7.07(25.28-51.45)$ & $29.97 \pm 7.54(25.33-51.32)$ & 0.779 \\
\hline Gender (male:female) & $18: 2(90 \%: 10 \%)$ & $19: 1(95 \%: 5 \%)$ & 0.000 \\
\hline Dominant side (right:left) & $20: 0(100 \%: 0 \%)$ & $20: 0(100 \%: 0 \%)$ & - \\
\hline Effected side (right:left) & $15: 5(75 \%: 25 \%)$ & $13: 7(65 \%: 35 \%)$ & 0.011 \\
\hline
\end{tabular}

NMES, neuromuscular electrical stimulation.

hours in the NMES group and 29.97 \pm 7.54 hours in the control group $(\mathrm{p}=0.779)$ (Table I).

There was no difference between the baseline and preoperative measurements in operated side knee flexion ROM in the NMES group ( $\mathrm{p}>0.05$ ). Postoperative operated side knee flexion ROM was significantly better than baseline and preoperative scores in both groups, with no difference between the groups $(\mathrm{p}<0.05)$. While there was no difference between the groups at baseline ( $>0.05)$, the operated side quadriceps muscle strength of the NMES group was significantly higher than the control group in the preoperative evaluation $(\mathrm{p}=0.028)$ There was no difference between the groups in the postoperative period $(\mathrm{p}>0.05)$ (Table II).

There was no statistically significant difference between NMES and control groups in terms of KOOS and WOMAC scores at baseline $(\mathrm{p}>0.05)$. KOOS pain $(p=0.004)$, other symptoms $(p=0.015)$, function in daily living $(p=0.0001)$ and WOMAC total score $(p=0.0001)$ of the NMES group was significantly higher than the control group at preoperative measurement. In within-group comparisons, pre-operative KOOS function in daily living and WOMAC total scores of the NMES group were statistically improved compared to the baseline $(p<0.05)$, while the other KOOS scores showed the tendency for an increase but were not significant $(\mathrm{p}>0.05)$ However postoperative KOOS and WOMAC scores were significantly better than baseline and preoperative scores in both groups, with no difference between groups (Table III).

Preoperative 30-second chair-stand test, 40-meter fast-paced walk test and stair-climb test scores of the NMES group tended to improve compared to baseline but were not statistically significant $(\mathrm{p}>0.05)$. However, the postoperative 12th week scores of all measurements in both groups were significantly better than at baseline $(\mathrm{p}<0.05)$. In between-group comparisons, only the 40-meter fast-paced walk test at the postoperative 12th week showed a difference in favor of the NMES group $(p=0.028)$ (Table IV).

There was no statistically significant difference between NMES and control groups in terms of WHOQOL-BREF scores at baseline. The WHOQOL-BREF Physical domain score of the NMES group was significantly higher than the control group at preoperative measurement $(\mathrm{p}<0.05)$, while there was a tendency to increase in other domains but did not reach statistical significance $(\mathrm{p}>0.05)$. However, with no difference between groups, postoperative WHOQOL-BREF Physical domain score was significantly higher than baseline and preoperative scores in both groups (Table V).

\section{DISCUSSION}

In this study, we evaluated the effects of preoperative six-week NMES application on the postoperative quadriceps muscle strength, functional status, and quality of life in fast track TKA patients. The most important finding of our study is that although preoperative NMES has beneficial effects in terms of patient-reported and performancebased physical functions and quality of life in the preoperative period, it has no additional benefit in postoperative outcomes.

Based on the evidence that postoperative functional recovery can be predicted with preoperative clinical and functional variables, preoperative physiotherapy interventions have 


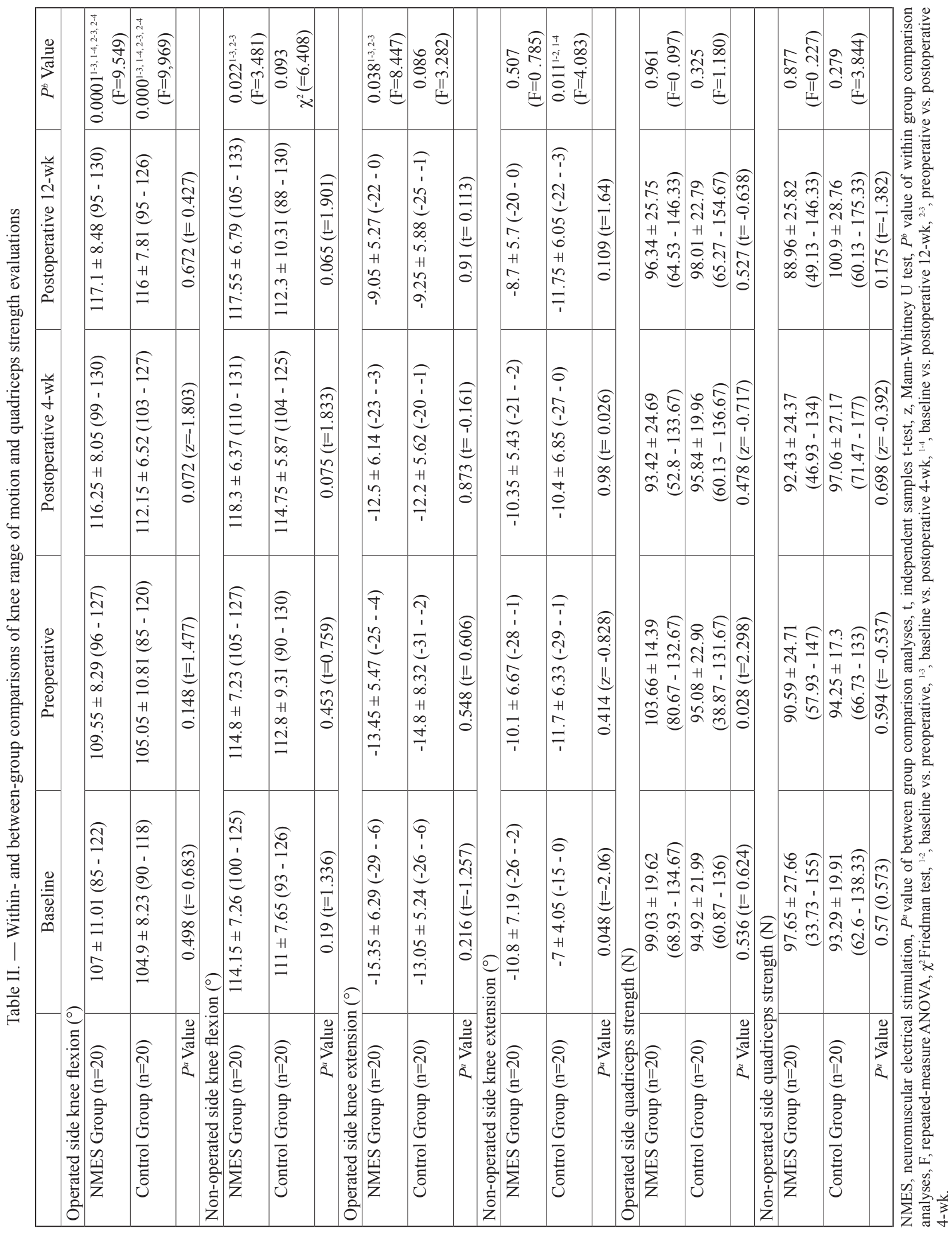


Table III. - Within- and between-group comparisons of patient reported activity limitation measurements.

\begin{tabular}{|c|c|c|c|c|c|}
\hline & Baseline & Preoperative & Postoperative 4-wk & Postoperative 12-wk & $P^{b}$ Value \\
\hline \multicolumn{6}{|l|}{ KOOS-Pain } \\
\hline NMES Group $(\mathrm{n}=20)$ & $\begin{array}{c}43.33 \pm 17.9 \\
(13.89-75) \\
\end{array}$ & $\begin{array}{l}53.06 \pm 17.93 \\
(16.67-100) \\
\end{array}$ & $\begin{array}{r}73.06 \pm 17.2 \\
(38.89-100) \\
\end{array}$ & $\begin{array}{c}83.89 \pm 15.2 \\
(50-100)\end{array}$ & $\begin{array}{c}0.0001^{1-3,1-4,2-3,2-4,3-4} \\
(\mathrm{~F}=35.639)\end{array}$ \\
\hline Control Group $(\mathrm{n}=20)$ & $\begin{array}{c}39.58 \pm 12.05 \\
(16.67-58.33) \\
\end{array}$ & $\begin{array}{c}37.08 \pm 15.35 \\
(13.89-69.44) \\
\end{array}$ & $\begin{array}{r}66.53 \pm 17.67 \\
(36.11-100) \\
\end{array}$ & $\begin{array}{c}86.39 \pm 12.96 \\
(66.67-100) \\
\end{array}$ & $\begin{array}{c}0.000^{1-3,1-4,2-3,2-4,3-4} \\
(\mathrm{~F}=57.729)\end{array}$ \\
\hline$P^{a}$ Value & $0.443(\mathrm{t}=0.777)$ & $0.004(\mathrm{t}=3.026)$ & $0.244(\mathrm{t}=1.184)$ & $0.841(\mathrm{z}=-0.219)$ & \\
\hline \multicolumn{6}{|l|}{ KOOS-other Symptoms } \\
\hline NMES Group (n=20) & $\begin{array}{c}44.82 \pm 21.25 \\
(10.71-71.43)\end{array}$ & $\begin{array}{c}55.54 \pm 15.26 \\
(35.71-85.71) \\
\end{array}$ & $\begin{array}{c}76.61 \pm 11.67 \\
(46.43-96.43) \\
\end{array}$ & $\begin{array}{l}81.61 \pm 12.49 \\
(57.14-100)\end{array}$ & $\begin{array}{c}0.0001^{1-3,1-4,2-3,2-4} \\
(\mathrm{~F}=31.588)\end{array}$ \\
\hline Control Group $(n=20)$ & $\begin{array}{c}41.07 \pm 13.73 \\
(21.43-75) \\
\end{array}$ & $\begin{array}{c}41.61 \pm 19.05 \\
(10.71-75) \\
\end{array}$ & $\begin{array}{c}73.39 \pm 14.35 \\
(50-100) \\
\end{array}$ & $\begin{array}{c}77.68 \pm 11.98 \\
(57.14-100) \\
\end{array}$ & $\begin{array}{c}0.000^{1-3,1-4,2-3,2-4} \\
(\mathrm{~F}=42.207)\end{array}$ \\
\hline$P^{a}$ Value & $0.512(\mathrm{t}=0.663)$ & $0.015(\mathrm{t}=2.552)$ & $0.442(\mathrm{t}=0.777)$ & $0.317(\mathrm{t}=1.015)$ & \\
\hline \multicolumn{6}{|c|}{ KOOS-Function in daily living } \\
\hline NMES Group $(\mathrm{n}=20)$ & $\begin{array}{c}50.51 \pm 15.63 \\
(20.59-82.35)\end{array}$ & $\begin{array}{c}61.47 \pm 15.89 \\
(27.94-92.65) \\
\end{array}$ & $\begin{array}{c}83.68 \pm 13.22 \\
(51.47-98.53) \\
\end{array}$ & $\begin{array}{l}89.85 \pm 12.35 \\
(63.24-100) \\
\end{array}$ & $\begin{array}{c}0.0001^{1-2,1-3,1-4,2-3,2-4} \\
(\mathrm{~F}=41.715)\end{array}$ \\
\hline Control Group $(\mathrm{n}=20)$ & $\begin{array}{c}42.43 \pm 13.06 \\
(14.71-72.06) \\
\end{array}$ & $\begin{array}{c}42.65 \pm 11.31 \\
(19.12-61.76)\end{array}$ & $\begin{array}{c}73.53 \pm 17.76 \\
(39.71-97.06)\end{array}$ & $\begin{array}{l}87.21 \pm 11.19 \\
(70.59-100)\end{array}$ & $\begin{array}{c}0.000^{1-3,1-4,2-3,2-4,3-4} \\
(\mathrm{~F}=65.215)\end{array}$ \\
\hline$P^{a}$ Value & $0.084(\mathrm{t}=1.776)$ & $0.0001(\mathrm{t}=4.317)$ & $0.076(\mathrm{z}=-1.788)$ & $0.369(\mathrm{z}=-0.91)$ & \\
\hline \multicolumn{6}{|c|}{ KOOS-Function in sport and recreation } \\
\hline NMES Group $(\mathrm{n}=20)$ & $\begin{array}{c}2.25 \pm 5.25 \\
(0-20) \\
\end{array}$ & $\begin{array}{c}4 \pm 15.61 \\
(0-70)\end{array}$ & $\begin{array}{c}18.25 \pm 15.41 \\
(0-50)\end{array}$ & $\begin{array}{c}30.5 \pm 20.89 \\
(0-85)\end{array}$ & $\begin{array}{c}0.0001^{1-3,1-4,2-3,2-4} \\
\chi^{2}(=36.3)\end{array}$ \\
\hline Control Group $(\mathrm{n}=20)$ & $\begin{array}{c}2.5 \pm 5.26 \\
(0-15)\end{array}$ & $\begin{array}{c}3.25 \pm 7.12 \\
(0-25)\end{array}$ & $\begin{array}{c}18 \pm 12.4 \\
(0-40)\end{array}$ & $\begin{array}{c}23.75 \pm 21.82 \\
(0-90)\end{array}$ & $\begin{array}{c}0.000^{1-3,1-4,2-3,2-4} \\
\chi^{2}(=39.786)\end{array}$ \\
\hline$P^{a}$ Value & $0.968(\mathrm{z}=-0.078)$ & $0.758(z=-0.49)$ & $0.883(\mathrm{z}=-0.15)$ & $0.192(\mathrm{z}=-1.339)$ & \\
\hline \multicolumn{6}{|c|}{ KOOS-Knee-related quality of life } \\
\hline NMES Group (n=20) & $\begin{array}{c}25.63 \pm 20.57(0 \\
-62.5)\end{array}$ & $\begin{array}{c}33.75 \pm 20.22 \\
(0-62.5)\end{array}$ & $\begin{array}{c}62.81 \pm 23.95 \\
(25-93.75)\end{array}$ & $\begin{array}{c}73.75 \pm 23.61 \\
(25-100) \\
\end{array}$ & $\begin{array}{c}0.0001^{1-3,1-4,2-3,2-4} \\
(\mathrm{~F}=29.119)\end{array}$ \\
\hline Control Group $(\mathrm{n}=20)$ & $\begin{array}{c}23.12 \pm 19.67(0- \\
68.75)\end{array}$ & $\begin{array}{c}23.13 \pm 21.47 \\
(0-68.75)\end{array}$ & $\begin{array}{l}54.06 \pm 26.54 \\
(12.5-93.75)\end{array}$ & $\begin{array}{c}68.44 \pm 26.4 \\
(18.75-100) \\
\end{array}$ & $\begin{array}{l}0.000^{1-3,1-4,2-3,2-4} \\
\chi^{2}(=29.231)\end{array}$ \\
\hline$P^{a}$ Value & $0.734(\mathrm{z}=-0.340)$ & $0.114(z=-1.599)$ & $0.281(\mathrm{t}=1.095)$ & $0.547(z=-0.614)$ & \\
\hline \multicolumn{6}{|l|}{ WOMAC-Total } \\
\hline NMES Group (n=20) & $\begin{aligned} 49.35 & \pm 13.57(25 \\
& -72)\end{aligned}$ & $\begin{array}{c}39.6 \pm 13.74 \\
(16-67)\end{array}$ & $\begin{array}{c}19.95 \pm 12.17 \\
(3-53)\end{array}$ & $\begin{array}{c}12.3 \pm 10.15 \\
(0-32)\end{array}$ & $\begin{array}{c}0.0001^{1-2,1-3,1-4,2-3,2-4} \\
(\mathrm{~F}=47.255)\end{array}$ \\
\hline Control Group $(n=20)$ & $\begin{array}{c}56.25 \pm 11.36(26 \\
-79)\end{array}$ & $\begin{array}{c}57.25 \pm 11.28 \\
(39-80)\end{array}$ & $\begin{array}{c}24.35 \pm 13.16 \\
(5-55)\end{array}$ & $\begin{array}{c}15.25 \pm 12.17 \\
(0-43)\end{array}$ & $\begin{array}{c}0.000^{1-3,1-4,2-3,2-4,3-4} \\
(\mathrm{~F}=75.306)\end{array}$ \\
\hline$P^{a}$ Value & $0.089(\mathrm{t}=-1.743)$ & $0.0001(\mathrm{t}=-4.441)$ & $0.279(\mathrm{t}=-1.098)$ & $0.41(\mathrm{t}=-0.832)$ & \\
\hline
\end{tabular}

NMES, neuromuscular electrical stimulation, $P^{a}$ value of between group comparison analyses, $\mathrm{t}$, independent samples $\mathrm{t}$-test, $\mathrm{z}$, Mann-Whitney U test, $P^{b}$ value of within group comparison analyses, F, repeated-measure ANOVA, $\chi^{2}$ Friedman test, ${ }^{1-2}$, baseline vs. preoperative, ${ }^{1-3}$, baseline vs. postoperative $4-\mathrm{wk},{ }^{1-4}$, baseline vs. postoperative $12-\mathrm{wk},{ }^{2-3}$, preoperative vs. postoperative $4-\mathrm{wk},{ }^{2-4}$, preoperative vs. postoperative $12-w k,{ }^{3-4}$, postoperative 4 -wk vs. postoperative 12 -wk.

recently attracted the attention of researchers. However, interventions generally focused on patient education and exercise therapy $(14,15)$, to our knowledge only one pilot study investigated the effect of NMES (10). Systematic reviews and metaanalyzes examining the effectiveness of exercise therapy showed that preoperative physiotherapy did not provide significant postoperative benefit in key outcomes such as function, pain, quality of life, and length of stay $(16,17)$. Although NMES has a potential role in accelerating postoperative recovery and improving functionality (11), making a decision based on only one study result is difficult. We examined the effectiveness of preoperative NMES 
EFFECTS OF PREOPERATIVE NEUROMUSCULAR ELECTRICAL STIMULATION ON THE POSTOPERATIVE OUTCOMES

Table IV. - Within- and between-group comparisons of performance-based activity limitation measurements

\begin{tabular}{|c|c|c|c|c|c|}
\hline & Baseline & Preoperative & Postoperative 4-wk & Postoperative 12-wk & $P^{b}$ Value \\
\hline \multicolumn{6}{|l|}{ 30-second chair-stand test } \\
\hline NMES Group $(\mathrm{n}=20)$ & $\begin{array}{c}8.3 \pm 2.031 \\
(3-13)\end{array}$ & $\begin{array}{c}9.75 \pm 1.86 \\
(6-13)\end{array}$ & $10 \pm 2.22(6-16)$ & $11.4 \pm 2.14(8-16)$ & $\begin{array}{c}0.0001^{1-4} \\
\left(\chi^{2}=22.95\right)\end{array}$ \\
\hline Control Group $(\mathrm{n}=20)$ & $\begin{array}{c}8.85 \pm 1.98 \\
(5-12)\end{array}$ & $\begin{array}{c}9.8 \pm 2.14 \\
(6-14)\end{array}$ & $9.95 \pm 2.14(6-16)$ & $11.6 \pm 2.3(7-17)$ & $\begin{array}{l}0.000^{1-4,2-4,3-4} \\
\left(\chi^{2}=33.074\right)\end{array}$ \\
\hline$P^{a}$ Value & $0.341(\mathrm{z}=-1)$ & $0.938(\mathrm{t}=-0.079)$ & $0.943(\mathrm{t}=0.072)$ & $0.777(\mathrm{t}=-0.285)$ & \\
\hline \multicolumn{6}{|c|}{ 40-meter fast-paced walk test } \\
\hline NMES Group $(\mathrm{n}=20)$ & $\begin{array}{c}42.27 \pm 18 \\
(27.62-87.71)\end{array}$ & $\begin{array}{c}39.7 \pm 11.54 \\
(27.13-70.08) \\
\end{array}$ & $\begin{array}{c}38.07 \pm 8.94 \\
(25.89-59.75) \\
\end{array}$ & $\begin{array}{c}34.66 \pm 8.41 \\
(24.94-60.73) \\
\end{array}$ & $\begin{array}{l}0.002^{1-4,2-4,3-4} \\
\left(\chi^{2}=14.46\right)\end{array}$ \\
\hline Control Group $(\mathrm{n}=20)$ & $\begin{array}{l}44.29 \pm 10.71 \\
(26.44-69.29) \\
\end{array}$ & $\begin{array}{c}45.24 \pm 12.44 \\
(33.93-91.87) \\
\end{array}$ & $\begin{array}{c}41.91 \pm 10.15 \\
(26.21-75.47) \\
\end{array}$ & $\begin{array}{c}38.92 \pm 7.42 \\
(22.4-56.43) \\
\end{array}$ & $\begin{array}{c}0.034^{1-4,2,4} \\
\left(\chi^{2}=8.698\right)\end{array}$ \\
\hline$P^{a}$ Value & $0.114(\mathrm{z}=-1.596)$ & $0.056(z=-1.921)$ & $0.149(z=-1.461)$ & $0.028(\mathrm{z}=-2.191)$ & \\
\hline \multicolumn{6}{|l|}{ Stair-climb test } \\
\hline NMES Group $(\mathrm{n}=20)$ & $\begin{array}{c}25.7 \pm 9.81 \\
(13.22-48.73) \\
\end{array}$ & $\begin{array}{c}23.99 \pm 10.82 \\
(11.74-49.79) \\
\end{array}$ & $\begin{array}{c}24.36 \pm 9.41 \\
(12.4-44.63) \\
\end{array}$ & $\begin{array}{c}18.88 \pm 6.59 \\
(10.26-31.19) \\
\end{array}$ & $\begin{array}{c}0.001^{1-4,2-4,3-4} \\
(\mathrm{~F}=6.374)\end{array}$ \\
\hline Control Group $(\mathrm{n}=20)$ & $\begin{array}{c}28.28 \pm 8.55 \\
(18.02-47.87) \\
\end{array}$ & $\begin{array}{c}28.61 \pm 8.3 \\
(17.33-54.89) \\
\end{array}$ & $\begin{array}{c}25.41 \pm 7.33 \\
(11.15-46.97) \\
\end{array}$ & $\begin{array}{c}22.43 \pm 7.35 \\
(10.23-38.19)\end{array}$ & $\begin{array}{c}0.002^{1-4,2-4} \\
\left(\chi^{2}=14.820\right)\end{array}$ \\
\hline$P^{a}$ Value & $0.165(\mathrm{z}=-1.407)$ & $0.072(\mathrm{z}=-1.812)$ & $0.696(\mathrm{t}=-0.394)$ & $0.117(\mathrm{t}=-1.605)$ & \\
\hline
\end{tabular}

NMES, neuromuscular electrical stimulation, $P^{a}$ value of between group comparison analyses, $\mathrm{t}$, independent samples $\mathrm{t}$-test, $\mathrm{z}$, Mann-Whitney $\mathrm{U}$ test, $P^{b}$ value of within group comparison analyses, F, repeated-measure ANOVA, $\chi^{2}$ Friedman test, ${ }^{1-4}$, baseline vs. postoperative $12-\mathrm{wk},{ }^{2-4}$, preoperative vs. postoperative $12-\mathrm{wk},{ }^{3-4}$, postoperative 4 -wk vs. postoperative 12 -wk.

in patients undergoing fast-track TKA with the idea that obtaining postoperative enhanced recovery can be achieved with multimodal approaches. The most important finding of the present study is that preoperative NMES does not provide additional benefit in terms of postoperative outcome measurements.

The pain of knee osteoarthritis may cause functional capacity decline and muscle atrophy by reflex mechanisms (18). Meta-analyzes has reported that NMES is not appropriate for pain relief in patients with knee osteoarthritis (19) or evidence of its effect on pain is inconsistent (20). Our finding was consistent with a previous study (10) that the preoperative NMES had no effect on preoperative pain relief and postoperative outcomes following TKA surgery. Also, NMES did not have an effect on knee flexion range in patients with stage IV knee osteoarthritis in our study.

Compared to age-matched healthy peers, TKA patients walk slower and have more difficulty climbing stairs and performing daily living activities (21). Quadriceps muscle weakness may persist longer than 3 years postoperatively in these patients (22). Although we planned this study with the knowledge that optimizing the preoperative quadriceps muscle strength plays a dominant role in achieving enhanced functional outcomes (12), we did not find a statistically significant increase in quadriceps muscle strength with NMES application. Further, results in the systematic review regarding the effect of NMES on quadriceps muscle strength in knee osteoarthritis alone or in combination with other therapeutic interventions are inconsistent (20), and a Cochrane review did not reach any conclusions about the application of NMES to strengthen quadriceps before or after TKA (23). Despite the lack of consensus on the optimal NMES parameter (24), Glaviano et al. (25) suggest that NMES, with a pulse duration of 200 to 400 microseconds delivered at 30 to $50 \mathrm{~Hz}$ at the highest tolerated intensity, can be used to strengthen the quadriceps muscle. We referenced this study for the intensity of NMES, but the absence of a significant strength increase may be due to our stimulation intensity not reaching a sufficient level to strengthen the muscle. Therefore, further research is needed to determine the optimal NMES intensity in muscle strengthening. Also, the 
Table V. - Within- and between-group comparisons of the quality of life measurements

\begin{tabular}{|c|c|c|c|c|c|}
\hline & Baseline & Preoperative & Postoperative 4-wk & Postoperative 12-wk & $P^{b}$ Value \\
\hline \multicolumn{6}{|l|}{ WHOQOL-BREF Physical } \\
\hline NMES Group $(n=20)$ & $\begin{array}{c}49.82 \pm 20.65 \\
(10.71-82.14)\end{array}$ & $\begin{array}{l}60.71 \pm 19.11 \\
(35.71-100)\end{array}$ & $\begin{array}{l}75.54 \pm 14.16 \\
(53.57-100)\end{array}$ & $\begin{array}{c}79.29 \pm 16 \\
(46.43-100)\end{array}$ & $\begin{array}{c}0.0001^{1-3,1-4,2-3,2-4} \\
(\mathrm{~F}=16.851)\end{array}$ \\
\hline Control Group $(n=20)$ & $\begin{array}{c}45.71 \pm 18.49 \\
(14.29-85.71)\end{array}$ & $\begin{array}{c}42.5 \pm 22.04 \\
(3.57-82.14)\end{array}$ & $\begin{array}{c}63.39 \pm 20.53 \\
(28.57-96.43)\end{array}$ & $\begin{array}{l}75.36 \pm 17.87 \\
(46.43-100)\end{array}$ & $\begin{array}{c}0.000^{1-3,1-4,2-3,2-4} \\
(\mathrm{~F}=18.292)\end{array}$ \\
\hline$P^{a}$ Value & $0.511(\mathrm{t}=0.663)$ & $0.008(\mathrm{t}=2.792)$ & $0.036(\mathrm{t}=2.178)$ & $0.468(\mathrm{t}=0.732)$ & \\
\hline \multicolumn{6}{|c|}{ WHOQOL-BREF Psychologic } \\
\hline NMES Group $(\mathrm{n}=20)$ & $\begin{array}{c}71.04 \pm 20.39 \\
(41.67-100)\end{array}$ & $\begin{array}{l}80.83 \pm 20.52 \\
(33.33-100)\end{array}$ & $\begin{array}{c}83.75 \pm 15.58 \\
(45.83-100)\end{array}$ & $\begin{array}{c}82.92 \pm 17.25 \\
(50-100)\end{array}$ & $\begin{array}{c}0.008^{1-4} \\
(\mathrm{~F}=4.363)\end{array}$ \\
\hline Control Group $(\mathrm{n}=20)$ & $\begin{array}{c}65.83 \pm 23.98 \\
(8.33-100)\end{array}$ & $\begin{array}{c}66.67 \pm 27.74 \\
(0-100)\end{array}$ & $\begin{array}{l}72.5 \pm 16.85 \\
(45.83-100)\end{array}$ & $\begin{array}{l}73.75 \pm 18.09 \\
(45.83-100)\end{array}$ & $\begin{array}{c}0.604 \\
\left(\chi^{2}=1.85\right)\end{array}$ \\
\hline$P^{a}$ Value & $0.464(\mathrm{t}=0.74)$ & $0.081(\mathrm{z}=-1.771)$ & $0.033(\mathrm{z}=-2.133)$ & $0.096(\mathrm{z}=-1.677)$ & \\
\hline \multicolumn{6}{|l|}{ WHOQOL-BREF Social } \\
\hline NMES Group (n=20) & $\begin{array}{c}78.33 \pm 16.75 \\
(50-100)\end{array}$ & $\begin{array}{l}85 \pm 24.57 \\
(25-100)\end{array}$ & $\begin{array}{c}85.83 \pm 24.79 \\
(16.67-100)\end{array}$ & $\begin{array}{c}86.67 \pm 19.19 \\
(41.67-100)\end{array}$ & $\begin{array}{c}0.298 \\
\left(\chi^{2}=3.68\right)\end{array}$ \\
\hline Control Group $(n=20)$ & $\begin{array}{l}87.08 \pm 21.37 \\
(16.67-100)\end{array}$ & $\begin{array}{l}90 \pm 13.68( \\
66.67-100)\end{array}$ & $\begin{array}{l}79.38 \pm 17.28 \\
(41.67-100)\end{array}$ & $\begin{array}{c}80.21 \pm 19.63 \\
(33.33-100)\end{array}$ & $\begin{array}{c}0.182 \\
\left(\chi^{2}=4.860\right)\end{array}$ \\
\hline$P^{a}$ Value & $0.072(\mathrm{z}=-1.919)$ & $0.947(\mathrm{z}=-0.079)$ & $0.086(\mathrm{z}=-1.834)$ & $0.253(\mathrm{z}=-1.235)$ & \\
\hline \multicolumn{6}{|c|}{ WHOQOL-BREF Environment } \\
\hline NMES Group $(\mathrm{n}=20)$ & $\begin{array}{c}81.11 \pm 15.73 \\
(50-100)\end{array}$ & $\begin{array}{c}86.81 \pm 21.17 \\
(27.78-100)\end{array}$ & $\begin{array}{l}87.92 \pm 15.03 \\
(47.22-100)\end{array}$ & $\begin{array}{c}87.97 \pm 16.45 \\
(40.63-100)\end{array}$ & $\begin{array}{c}0.148 \\
(\mathrm{~F}=1.855)\end{array}$ \\
\hline Control Group $(\mathrm{n}=20)$ & $\begin{array}{c}86.94 \pm 17.48 \\
(25-100)\end{array}$ & $\begin{array}{c}84.44 \pm 15.9 \\
(47.22-100)\end{array}$ & $\begin{array}{c}83.75 \pm 15.51 \\
(41.67-100)\end{array}$ & $\begin{array}{c}81.41 \pm 16.14 \\
(53.13-100)\end{array}$ & $\begin{array}{c}0.632 \\
(\mathrm{~F}=0.577)\end{array}$ \\
\hline$P^{a}$ Value & $0.142(\mathrm{z}=-1.481)$ & $0.289(\mathrm{z}=-1.119)$ & $0.341(\mathrm{z}=-0.992)$ & $0.201(\mathrm{z}=-1.323)$ & \\
\hline
\end{tabular}

NMES, neuromuscular electrical stimulation, $P^{a}$ value of between group comparison analyses, $t$, independent samples $\mathrm{t}$-test, $\mathrm{z}$, Mann-Whitney U test, $P^{b}$ value of within group comparison analyses, F, repeated-measure ANOVA, $\chi^{2}$ Friedman test, ${ }^{1-3}$, baseline vs. postoperative $4-\mathrm{wk},{ }^{1-4}$, baseline vs. postoperative $12-\mathrm{wk},{ }^{2-3}$, preoperative vs. postoperative $4-\mathrm{wk},{ }^{2-4}$, preoperative vs. postoperative 12-wk.

use of a fast-track protocol, including procedures to minimize surgical trauma, may have prevented atherogenic muscle inhibition from surgical trauma.

Knee osteoarthritis causes pain, limited ROM, and muscle weakness, leading to a limitation in daily living activities. The use of NMES is recommended to improve functionality in patients with knee osteoarthritis who do not participate in any exercise program, are contraindicated to exercise, or do not like physical activity (26). There are conflicting results concerning the effectiveness of NMES application on physical functions in knee osteoarthritis. Whereas some studies reported a significant improvement in WOMAC total score with NMES (27), other studies have failed to find difference $(28,29)$. We found a statistically significant improvement in KOOS-function in daily living and WOMAC total score after NMES application at preoperative evaluation. Although NMES had beneficial effects on preoperative patient-reported physical functions, there was no difference between the groups in the postoperative period.

The recovery of locomotor abilities is one of the main therapeutic goals of knee osteoarthritis because most patients have difficulty in walking and stair negotiation, which reflects all physical functions (30). Patients with knee osteoarthritis who have stronger quadriceps and hamstring muscles and less knee pain have the better stair-climbing ability (31). The walking, stair-climbing and chair rise ability can be improved with NMES $(10,32)$. We also found a trend towards an increase in performance-based activity, although it was not statistically significant. The reason for the inconsistency between the results of patient- and performance-based activity limitations may be that self-reports of physical function do not 
always correlate closely with objective measures of the ability to perform activities (33). Although there was no difference between the groups in preoperative period, the NMES group had a faster walking pace at the postoperative 12th week. Therefore, NMES may have a positive effect on the walking pace of patients in the postoperative period.

Some researchers suggest that concept of healthrelated quality of life is a sub-component of generic quality of life, so it is impossible to distinguish between these two concepts. The quality of life was measured with the WHOQOL-BREF scale, and we found a difference between the groups in favor of the NMES group only in preoperative measurement. This may be because improvements in patient-reported physical functions after NMES application have a positive effect on the physical health domain of quality of life. However, since there is no difference between the groups in the postoperative period, it has an advantage only for a short time.

This study has some limitations. One of the possible limitations of our study was that NMES stimulation intensity was not recorded. The intensity of stimulation was "maximum tolerated strong visible muscle contraction by the patient" as indicated in previous studies $(10,24,26,34)$. Differences such as OA stage, sample size and NMES dosage in the studies led to the lack of conclusive judgment on the dose-response effect. Maximum voluntary contraction can be measured to determine the optimal dosage of NMES in further studies. Besides, we did not measure the cross-sectional area and voluntary activation of the quadriceps muscle. Recent evidence supports that quadriceps weakness in stage IV knee osteoarthritis is more predominantly attributed to a failure in quadriceps voluntary muscle activation than to muscle atrophy (28). The effect of NMES on the cross-sectional area of the muscle and the reflection of these changes are not clearly known (31). In future studies, measuring quadriceps muscle strength as well as crosssectional area and voluntary activation will allow a more comprehensive determination of the effect of NMES on the neural and structural features of the muscle. Another limitation of our study is that we evaluated quadriceps muscle strength with a hand- held dynamometer. Although the hand dynamometer is often preferred in the clinic due to its portable, low cost and easy use, its reliability in measuring lower extremity strength varies among the authors.

\section{CONCLUSION}

In this study, based on the idea that improved postoperative enhanced recovery can be achieved with multimodal approaches; we examined the effectiveness of preoperative NMES application on postoperative quadriceps muscle strength, functional status, and quality of life outcomes in patients with fast-track TKA. According to our study results, preoperative NMES application has positive effects on the patient-reported physical functions and quality of life in the preoperative period, but it does not provide an additional benefit in terms of postoperative outcomes.

\section{REFERENCES}

1. Dillon CF, Rasch EK, Gu Q, Hirsch R. Prevalence of knee osteoarthritis in the United States: arthritis data from the Third National Health and Nutrition Examination Survey 1991-94. J Rheumatol. 2006 ; 33(11) : 2271-9.

2. Kamaruzaman H, Kinghorn P, Oppong R. Costeffectiveness of surgical interventions for the management of osteoarthritis : a systematic review of the literature. BMC Musculoskelet Disord. 2017 ; 18(1) : 183.

3. Kehlet H. Fast-track hip and knee arthroplasty. Lancet. $2013 ; 381(9878)$ : 1600-2.

4. White JJ, Houghton-Clemmey R, Marval P. Enhanced recovery after surgery (ERAS) : an orthopaedic perspective. $J$ Perioper Pract. 2013 Oct ; 23(10) : 228-32.

5. Husted H. Fast-track hip and knee arthroplasty : clinical and organizational aspects. Acta Orthop Suppl. 2012 ; 83(346) : 1-39.

6. Stevens JE, Mizner RL, Snyder-Mackler L. Quadriceps strength and volitional activation before and after total knee arthroplasty for osteoarthritis. J Orthop Res. 2003 ; 21(5) : 775-9.

7. Huang CH, Cheng CK, Lee YT, Lee KS. Muscle strength after successful total knee replacement: a 6- to 13-year followup. Clin Orthop Relat Res. 1996 ; (328) : 147-54.

8. Mizner RL, Petterson SC, Stevens JE, Axe MJ, SnyderMackler L. Preoperative quadriceps strength predicts functional ability one year after total knee arthroplasty. $J$ Rheumatol. 2005 ; 32(8) : 1533-9.

9. Yue C, Zhang X, Zhu Y, Jia Y, Wang H, Liu Y. Systematic review of three electrical stimulation techniques for rehabilitation after total knee arthroplasty. J Arthroplasty. $2018 ; 33(7): 2330-7$. 
10. Walls RJ, McHugh G, O'Gorman DJ, Moyna NM, O'Byrne JM. Effects of preoperative neuromuscular electrical stimulation on quadriceps strength and functional recovery in total knee arthroplasty. A pilot study. $B M C$ Musculoskelet Disord. $2010 ; 11: 119$.

11. Ibrahim MS, Khan MA, Nizam I, Haddad FS. Perioperative interventions producing better functional outcomes and enhanced recovery following total hip and knee arthroplasty : an evidence-based review. BMC Med. $2013 ; 11: 37$.

12. Alnahdi AH. Outcome measures capturing ICF domains in patient with total knee arthroplasty. Int $J$ Rehabil Res. 2014 ; 37(4) : 281-9.

13. Dobson F, Hinman RS, Roos EM, Abbott JH, Stratford P, Davis AM, et al. OARSI recommended performancebased tests to assess physical function in people diagnosed with hip or knee osteoarthritis. Osteoarthritis Cartilage. $2013 ; 21(8): 1042-52$.

14. McKay C, Prapavessis H, Doherty T. The effect of a prehabilitation exercise program on quadriceps strength for patients undergoing total knee arthroplasty : a randomized controlled pilot study. PM R. 2012 ; 4(9) : 647-56.

15. Beaupre LA, Lier D, Davies DM, Johnston DB. The effect of a preoperative exercise and education program on functional recovery, health related quality of life, and health service utilization following primary total knee arthroplasty. J Rheumatol. 2004 ; 31(6) : 1166-73.

16. Chesham RA, Shanmugam S. Does preoperative physiotherapy improve postoperative, patient-based outcomes in older adults who have undergone total knee arthroplasty? A systematic review. Physiother Theory Pract. 2017 ; 33(1) : 9-30.

17. Wang L, Lee M, Zhang Z, Moodie J, Cheng D, Martin J. Does preoperative rehabilitation for patients planning to undergo joint replacement surgery improve outcomes? A systematic review and meta-analysis of randomised controlled trials. BMJ Open. 2016 ; 6(2) : e009857.

18. Tok F, Aydemir K, Peker F, Safaz I, Taşkaynatan MA, Ozgül A. The effects of electrical stimulation combined with continuous passive motion versus isometric exercise on symptoms, functional capacity, quality of life and balance in knee osteoarthritis : randomized clinical trial. Rheumatol Int. $2011 ; 31(2)$ : 177-81.

19. Zeng C, Li H, Yang T, Deng ZH, Yang Y, Zhang $\mathbf{Y}$, et al. Electrical stimulation for pain relief in knee osteoarthritis: systematic review and network metaanalysis. Osteoarthritis Cartilage. 2015 ; 23(2) : 189-202.

20. Giggins O, Fullen B, Coughlan G. Neuromuscular electrical stimulation in the treatment of knee osteoarthritis : a systematic review and meta-analysis. Clin Rehabil. $2012 ; 26(10): 867-81$.

21. Noble PC, Gordon MJ, Weiss JM, Reddix RN, Conditt MA, Mathis KB. Does total knee replacement restore normal knee function? Clin Orthop Relat Res. 2005 ; (431) : 157-65.
22. Schache MB, McClelland JA, Webster KE. Lower limb strength following total knee arthroplasty: a systematic review. Knee. 2014 ; 21(1) : 12-20.

23. Monaghan B, Caulfield B, O'Mathúna DP. Surface neuromuscular electrical stimulation for quadriceps strengthening pre and post total knee replacement. Cochrane Database Syst Rev. 2010 ; 2010(1) : CD007177.

24. Imoto AM, Peccin MS, Teixeira LE, Silva KN, Abrahão M, Trevisani VF. Is neuromuscular electrical stimulation effective for improving pain, function and activities of daily living of knee osteoarthritis patients? A randomized clinical trial. Sao Paulo Med J. 2013 ; 131(2) : 80-7.

25. Glaviano NR, Saliba S. Can the Use of neuromuscular electrical stimulation be improved to optimize quadriceps strengthening? Sports Health. $2016 ; 8(1): 79-85$.

26. Rosemffet MG, Schneeberger EE, Citera G, Sgobba ME, Laiz C, Schmulevich H, et al. Effects of functional electrostimulation on pain, muscular strength, and functional capacity in patients with osteoarthritis of the knee. J Clin Rheumatol. 2004 ; 10(5) : 246-9.

27. Dadalto TV, de Souza CP, da Silva EB. Neuromuscular electrical stimulation, exercises against resistance, muscle strength, pain, and motor function in patients with primary osteoarthritis of the knee. Fisioter Mov. 2013 ; 26(4) : 777-89.

28. Elboim-Gabyzon M, Rozen N, Laufer Y. Does neuromuscular electrical stimulation enhance the effectiveness of an exercise programme in subjects with knee osteoarthritis? A randomized controlled trial. Clin Rehabil. 2013 ; 27(3) : 246-57.

29. Laufer Y, Shtraker H, Elboim Gabyzon M. The effects of exercise and neuromuscular electrical stimulation in subjects with knee osteoarthritis : a 3-month follow-up study. Clin Interv Aging. 2014 ; 9 : 1153-61.

30. Parent E, Moffet H. Preoperative predictors of locomotor ability two months after total knee arthroplasty for severe osteoarthritis. Arthritis Rheum. 2003 Feb 15; 49(1): 36-50.

31. Whitchelo T, McClelland JA, Webster KE. Factors associated with stair climbing ability in patients with knee osteoarthritis and knee arthroplasty : a systematic review. Disabil Rehabil. 2014 ; 36(13) : 1051-60.

32. Bruce-Brand RA, Walls RJ, Ong JC, Emerson BS, O'Byrne JM, Moyna NM. Effects of home-based resistance training and neuromuscular electrical stimulation in knee osteoarthritis : a randomized controlled trial. $B M C$ Musculoskelet Disord. $2012 ; 13$ : 118.

33. Stratford PW, Kennedy DM, Woodhouse LJ. Performance measures provide assessments of pain and function in people with advanced osteoarthritis of the hip or knee. Phys Ther. 2006 ; 86(11) : 1489-96.

34. Nussbaum EL, Houghton P, Anthony J, Rennie S, Shay BL, Hoens AM. Neuromuscular electrical stimulation for treatment of muscle impairment : Critical review and recommendations for clinical practice. Physiother Can. $2017 ; 69(5): 1-76$. 